\title{
The Role of Commitment in Repeated Games*
}

\author{
Ignacio García-Juradoł Julio González-Díaz \\ Published in Optimization (2006) 55, 541-553 \\ Published version available at http://www.taylorandfrancisgroup.com \\ DOI 10.1080/02331930600815991
}

\begin{abstract}
We model the role of commitment in noncooperative games by means of what we call unilateral commitments. We study their impact within the framework of repeated games with complete information. To do so, we revisit the main folk theorems for repeated games with complete information and check up to what extent the assumptions needed in the classic models can be relaxed in the model with unilateral commitments.
\end{abstract}

KEYWORDS: repeated games, unilateral commitments, delegation games, folk theorems, complete information.

\section{Introduction}

The impact of different kinds of commitments in noncooperative models has been widely studied in game theoretical literature. Most of the contributions within this issue have been devoted to study delegation models; situations in which the players are represented by agents who play on their behalf. The concept of delegation, as well as other approaches to the idea of commitment, was already discussed in Schelling (1960). There has been an extensive research in the topic of delegation; see, for instance, Vickers (1985), Fershtman (1985), Sklivas (1987), and, more recently, Fershtman et al. (1991) and Caillaud et al. (1995). The model we present here is specially close to that in Fershtman et al. (1991). What they do is the following. To each 2-player strategic game, they associate a delegation game in which the agents play the original game on behalf of their principals. There is a first stage in which each principal, simultaneously and independently, provides his agent with a compensation scheme. In the second stage, the compensation schemes become common knowledge and the agents play the original game and have the payoffs given by the compensation schemes. Their main result is a folk theorem which, roughly speaking, states that if the original game has a Nash equilibrium, then every Pareto optimal allocation can be achieved as a subgame perfect equilibrium of the delegation game.

In this paper we study another model with commitments, not far from that of delegation, but with several differences. We begin with a motivation and then we informally introduce

\footnotetext{
*We thank Ehud Kalai for helpful comments. This research was partially supported by the Spanish Ministry for Education and Science and FEDER through project BEC2002-04102-C02-02 and from the Xunta de Galicia under project PGIDT03PXIC20701PN.

†University of Santiago de Compostela

$\ddagger$ Corresponding author: Department of Statistics and OR, University of Santiago de Compostela, 15782, Santiago de Compostela, Spain. Phone number: +34981563100. Fax number: +34981597054. E-mail address: julkin@usc.es
} 
the model. There are many strategic situations in real life in which one of the players gets rid of some of his strategies: a department that makes the commitment of not hiring its own graduate students; a firm announcing a limited edition of a certain product; a party announcing, during the election campaign, that they are not going to make certain alliances regardless of the final result of the election;... We model these situations by what we call unilateral commitments (UC). This is not the first time that unilateral commitments are studied in literature (see, for instance, Faíña-Medín et al. (1998) and García-Jurado et al. (2000)). To each $n$-player strategic game we associate its UC-extension as follows. There is a first stage in which each player, simultaneously and independently, chooses a subset of his set of strategies, i.e., he makes a commitment. In the second stage, the commitments become common knowledge and the players play the original game with the restrictions given by the respective commitments.

After the latter (informal) presentation of our model, we can stress some similarities and differences with the model in Fershtman et al. (1991). We do not have principals and agents, i.e., we have the same players in the two stages of our game. Nonetheless, our model is very close to that of delegation; consider the UC-extension of a game in which the strategies of player 1 belong to $A_{1}$ and he commits to play only strategies in $A_{1}^{c}$. Suppose, for simplicity, that all the payoffs of the original game are positive. This situation can be seen as a delegation game in which the principal chooses the following compensation scheme for his agent: (i) if the agent plays a strategy within $A_{1}^{c}$, then he receives some fixed proportion of his principal's payoff and (ii) if he plays some strategy not in $A_{1}^{c}$ then he receives some fixed negative payoff. It is worth to mention that, as far as the model in Fershtman et al. (1991) is concerned, the compensation schemes are restricted to functions that are weakly monotonic on the payoff received by the principal. On the contrary, the compensation scheme we have defined to "imitate" our commitment can be non-monotonic and, moreover, it can depend not only on the payoffs but also on the specific strategies leading to them. Anyhow, the monotonicity assumption in Fershtman et al. (1991) responded to technical reasons and it seems natural that the principal can sign "non-monotonic contracts" with his agent if both of them agree upon. One more similarity between the two models is the following: in the delegation game, the contracts are public and have to be respected and, in our model, we take the same assumptions for the commitments.

From the discussion above, we can conclude that our model with unilateral commitments can be seen as a particular family of delegation games; a family in which only some specific compensation schemes are possible. Hence, in an economic situation in which there is room for the contracts needed for the delegation games, there is also room for the kind of commitments we define in this paper (always taking into account that we work within a complete information framework). Moreover, recall that in this paper we model $n$-player games and not only 2-player situations as it is common in the delegation games literature.

We devote this paper to study the implications of unilateral commitments within the framework of repeated games with complete information. In García-Jurado et al. (2000) it is shown that, if unilateral commitments are possible, no assumption is needed in order to get the Nash folk theorem; but there is no result concerning subgame perfection. Under a slightly different framework, we show that, when unilateral commitments are possible, it is easier to find both Nash and subgame perfect equilibria supporting the "cooperative" payoffs of the original game; indeed, most of the folk theorems do not need any assumption at all when unilateral commitments are considered. Differently, from the point of view of optimization theory, since the Pareto optimal allocations represent the social optima of the problem at hand, the results we present in this paper say that under mild assumptions such optima can be supported by both Nash and subgame perfect equilibria.

The structure of this paper is as follows. In Section 2 we introduce the background 
concepts, the definition of a new equilibrium concept for extensive games, and the definition of the model with unilateral commitments. In Section 3 we present some folk theorems for repeated games when unilateral commitments are possible. In addition, we compare our results with those without unilateral commitments. Finally, we conclude in Section 4.

\section{Notation}

A strategic game $G$ is a triplet $(N, A, \varphi)$, where:

- $N:=\{1, \ldots, n\}$ is the set of players,

- $A:=\prod_{i \in N} A_{i}$ and $A_{i}$ is the set of player $i$ 's strategies,

- $\varphi:=\left(\varphi_{1}, \ldots, \varphi_{n}\right)$ and $\varphi_{i}: A \rightarrow \mathbb{R}$ is the payoff function of player $i$.

We assume that, for each $i \in N$, the sets $A_{i}$ are compact topological spaces and the functions $\varphi_{i}$ are continuous. Let $a_{-i}$ be a strategy profile for the players in $N \backslash\{i\}$ and $A_{-i}$ the set of such profiles. For each $i \in N$ and each $a_{-i} \in A_{-i}$, let $\mu_{i}\left(a_{-i}\right):=$ $\max _{a_{i} \in A_{i}}\left\{\varphi_{i}\left(a_{-i}, a_{i}\right)\right\}$. Note that the $\mu_{i}$ functions are continuous. Also, for each $i \in N$, let $v_{i}:=\min _{a_{-i} \in A_{-i}}\left\{\mu_{i}\left(a_{-i}\right)\right\}$. The vector $v:=\left\{v_{1}, \ldots, v_{n}\right\}$ is the minimax payoff vector. Let $F$ be the set of feasible payoffs, $F:=\operatorname{co}\{\varphi(a): a \in A\}$. Now, for each $i \in N$, let $p_{-i} \in \operatorname{argmin}_{a_{-i} \in A_{-i}}\left\{\mu_{i}\left(a_{-i}\right)\right\}$.

To avoid confusion with the strategies of the repeated game, in what follows we refer to the strategies $a_{i} \in A_{i}$ and the strategy profiles $a \in A$ of the stage game as actions and action profiles, respectively.

Next, given a game $G=(N, A, \varphi)$, we define the repeated game $G(\delta, T)$; the $T$-fold repetition of $G$ with discount parameter $\delta \in(0,1]$. A history at stage $t \in\{1, \ldots, T\}$ is defined as follows:

i) for $t=1$, an element of $A^{0}=\{*\}$, where $*$ is any element not belonging to $\bigcup_{k \in \mathbb{N}} A^{k}$.

ii) for $t \in\{2, \ldots, T\}$, an element of $A^{t-1}$.

The set of all histories is $H:=\bigcup_{t=1}^{T} A^{t-1}$. In the repeated game we assume perfect monitoring, i.e., each player can choose his action in the current stage in the light of all actions taken by all players in all previous stages. Hence, let $G(\delta, T)$ be the triplet $\left(N, S, \varphi^{\delta, T}\right)$, where:

- The set of players $N$ remains the same.

- $S:=\prod_{i \in N} S_{i}$ is the set of strategy profiles, where $S_{i}:=A_{i}^{H}$, i.e., the set of mappings from $H$ to $A_{i}$. Let $\sigma=\left(\sigma_{1}, \ldots, \sigma_{n}\right) \in S$ and $h \in H$; then, we denote the action profile $\left(\sigma_{1}(h), \ldots, \sigma_{n}(h)\right)$ by $\sigma(h)$. A strategy profile $\sigma \in S$ recursively determines the sequence of action profiles $\pi(\sigma) \in A^{T}$ as follows: $\pi^{1}(\sigma):=\sigma(*)$ and, for each $t \in$ $\{2, \ldots, T\}, \pi^{t}(\sigma)=\sigma\left(\pi^{1}(\sigma), \ldots, \pi^{t-1}(\sigma)\right)$. We refer to $\pi(\sigma)$ as the path determined by $\sigma$.

- The payoff function $\varphi^{\delta, T}$ is defined as follows. Let $\sigma \in S$. Then, player $i$ 's payoff in $G(\delta, T)$ is his average discounted stage payoff:

$$
\varphi_{i}^{\delta, T}(\sigma):=\frac{1-\delta}{1-\delta^{T}} \sum_{t=1}^{T} \delta^{t-1} \varphi_{i}\left(\pi^{t}(\sigma)\right)
$$

\footnotetext{
${ }^{1}$ If there are no discounts $(i . e .$, if $\delta=1)$, we have $\varphi_{i}^{1, T}(\sigma):=(1 / T) \sum_{t=1}^{T} \varphi_{i}\left(\pi^{t}(\sigma)\right)$.
} 
Finally, recall that, from our definitions, we only use pure actions. If mixed actions are to be taken into account for a given game, then we just define a new game having them as pure actions. Hence, for the moment, we are implicitly assuming that, when working with mixed actions, they are observable; i.e., the players do not only observe the realization of a mixed action, but also the randomization process that leads to such a realization. We discuss a little bit more on the observability of mixed actions later on; indeed, we show that that assumption is not needed.

\subsection{Virtually Subgame Perfect Equilibrium}

A repeated game with perfect monitoring can be represented as an extensive game and, more specifically, as a multi-stage game with observed actions 2 Subgame perfect equilibrium (Selten, 1965), shortly SPE, is probably the most important equilibrium concept within this class of games. Its main target is to disregard those Nash equilibria which are only possible if some players give credit to irrational plans of others. More formally, a SPE is a Nash equilibrium which, moreover, induces a Nash equilibrium in every subgame 3

In this Section we introduce a new equilibrium concept for extensive games which is essential for this paper: the virtually subgame perfect equilibrium, shortly VSPE. This equilibrium concept has the same effect as subgame perfection, but it only concentrates on those subgames which are relevant for a given strategy profile; relevant in the sense that they are reachable if exactly one player deviates from the strategy profile in any subgame which has already been classified as relevant. Despite of being based on the same idea, SPE and VSPE are different concepts, the latter existing in many games which do not have SPE. Hence, VSPE is especially useful when dealing with extensive games having large trees. There are many extensive games without SPE, but still, they can have sensible equilibria. This is the case when the non-existence of SPE is because some subgames which are irrelevant for a certain strategy profile do not have Nash equilibria.

Let $\Gamma$ be an extensive game and $\sigma$ a strategy profile. Let $\Gamma_{x}$ denote the subgame of $\Gamma$ that begins at the single-node information set $x$. Let $\sigma_{x}$ be the restriction of $\sigma$ to $\Gamma_{x}$. Now, a subgame $\Gamma_{x}$ is $\sigma$-relevant if either (i) $\Gamma_{x}=\Gamma$, or (ii) there are a player $i$, a strategy $\sigma_{i}^{\prime}$, and a $\sigma$-relevant subgame $\Gamma_{y}$ such that node $x$ is reached by $\left(\sigma_{-i}, \sigma_{i}^{\prime}\right)_{y}$.

Definition 1. Let $\Gamma$ be an extensive game. The strategy profile $\sigma$ is a virtually subgame perfect equilibrium of $\Gamma$ if for each $\sigma$-relevant subgame $\Gamma_{x}$, then $\sigma_{x}$ is a Nash equilibrium of $\Gamma_{x}$.

Let $\operatorname{SPE}(\Gamma)$ and $\operatorname{VSPE}(\Gamma)$ denote the sets of SPE and VSPE of game $\Gamma$, respectively. By definition, for each extensive game $\Gamma$, we have $\operatorname{SPE}(\Gamma) \subseteq \operatorname{VSPE}(\Gamma)$. However, the reciprocal is not true as the following example illustrates.

Example 1. Consider the extensive game depicted in Figure 1 Let $\sigma=\left(\left(D_{1}, a_{1}^{i}\right),\left(D_{2}, a_{2}^{i}\right)\right)$, with $i \in\{1,2\}$. Clearly, since the subgame that begins after playing $\left(U_{1}, U_{2}\right)$ is not $\sigma$ relevant, $\sigma$ is a VSPE. However, this game does not have any SPE (in pure strategies). Moreover, the equilibrium $\sigma$ is a sensible one.

Next, we point out one more relation between SPE and VSPE. Let $\Gamma$ be an extensive game. Let $\sigma$ and $\hat{\sigma}$ be two strategy profiles of $\Gamma$. Now, let $\bar{\sigma}$ be the strategy profile which consists of playing in accordance with $\hat{\sigma}$ in all the subgames which are not $\sigma$-relevant and in accordance with $\sigma$ elsewhere. Then, the following statements hold:

\footnotetext{
${ }^{2}$ We model extensive games following the framework used in Kreps and Wilson (1982), except for the fact that we consider that the set of nodes may be infinite.

${ }^{3}$ Refer to van Damme (1991) for a deep analysis of the classic equilibrium concepts in $n$-person games.
} 


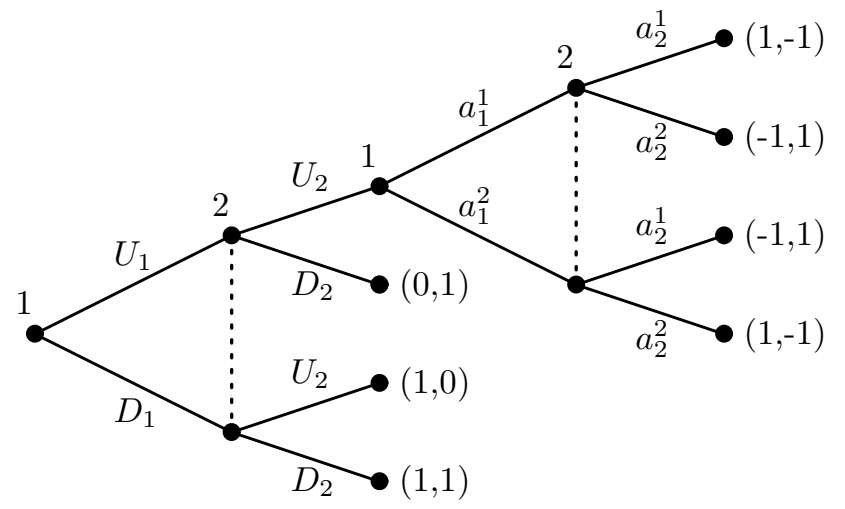

Figure 1: A game without SPE, but with VSPE.

i) The payoffs associated with $\sigma$ and $\bar{\sigma}$ coincide (they define the same path).

ii) If $\sigma \in \operatorname{VSPE}(\Gamma)$, then $\bar{\sigma} \in \operatorname{VSPE}(\Gamma)$.

iii) If $\sigma \in \operatorname{VSPE}(\Gamma)$ and $\hat{\sigma} \in \operatorname{SPE}(\Gamma)$, then $\bar{\sigma} \in \operatorname{SPE}(\Gamma)$.

Remark. In this paper we study a special family of multistage games with observed actions. The main reason why we need the concept of VSPE is the following: the extensive games we deal with in this paper are such that the actions available to the players at each node need not be convex sets. Hence, we cannot apply the classic results for the existence of subgame perfect equilibria.

\subsection{Unilateral Commitments}

The main objective of this paper is to study the effect of unilateral commitments on the appearing of constructive behavior in repeated games. Given a game $G$, the corresponding game with unilateral commitments consists of adding an initial stage to $G$; in this new stage each player can commit not to play certain strategies of game $G$. Moreover, these commitments are made simultaneously and unilaterally. The fact that the commitments have to be unilateral is quite important; if players could condition their commitments on the commitments of the others, then we would somehow be in a cooperative model, and hence, the players could easily achieve in equilibrium the cooperative payoffs of the game.

The problem of unilateral commitments, henceforth UC, has already been tackled in García-Jurado et al. (2000). They obtained a Nash folk theorem for finitely repeated games with UC. In this paper we deepen a little bit more in the impact of UC in the assumptions needed for the folk theorems. Next, following García-Jurado et al. (2000), we formally define the UC-extension of a game.

Given a game $G=(N, A, \varphi)$, we define the $U C$-extension of $G, U(G)$, as follows. There is a preliminary stage in which players choose, simultaneously and independently, a nonempty subset of their sets of strategies. Formally, each player $i \in N$ chooses $A_{i}^{c} \subseteq A_{i}$, where $A_{i}^{c}$ has to be a compact set. This election is interpreted as a commitment to play strategies only in $A_{i}^{c}$. Then, this preliminary stage ends and the commitments of the players, $A^{c}$, are made public, i.e., they become common knowledge. Finally, a reduced version of game $G$ in which players have to respect their commitments is played. Note that, as we have 
already pointed out, this kind of commitments are unilateral because we do not allow them to be conditional on the other players' commitments. The compactness assumption for the sets $A_{i}^{c}$ responds, as usually, to technical reasons; it ensures that the subgames starting after the stage of commitments belong to the class of games defined at the beginning of this Section. Note that, in the particular case in which the sets of strategies of the game under consideration are finite, the compactness requirement imposes no restriction at all. Throughout the rest of this Section, with a slight abuse of notation, given a set $A$, we use $2^{A}$ to denote the set of non-empty and compact subsets of $A$. Now, $U(G):=\left(N, A^{U}, \varphi^{U}\right)$, where:

- The set of players $N$ remains the same.

- $A^{U}:=\prod_{i \in N} A_{i}^{U}$, where $A_{i}^{U}$ is the set of all couples $\left(A_{i}^{c}, \alpha_{i}\right)$ such that

i) $A_{i}^{c} \subseteq A_{i}, A_{i}^{c} \neq \emptyset$,

ii) $\alpha_{i}: \prod_{j \in N} 2^{A_{j}} \longrightarrow A_{i}$ and, for each $A^{c} \in \prod_{j \in N} 2^{A_{j}}, \alpha_{i}\left(A^{c}\right) \in A_{i}^{c}$.

- The payoff associated with a strategy profile $\left(A^{c}, \alpha\right)$ is $\varphi^{U}\left(A^{c}, \alpha\right):=\varphi\left(\alpha\left(A^{c}\right)\right)$.

\section{The Folk Theorems}

The appearing of constructive behavior in repeated games has been widely treated in the game theoretical literature 4 Given a game $G$, the classical Nash folk theorem for finitely repeated games (Benoit and Krishna, 1987) states that if the game $G$ is such that, for each player $i$, there is a Nash equilibrum that gives $i$ something strictly better than his minimax payoff, then every feasible and individually rational payoff of $G$ can be approximated by a Nash equilibrium of $G(\delta, T)$ for big enough $T$ and $\delta$ close enough to 1 . Recently, GonzálezDíaz (2006) introduced a new condition, namely that the game $G$ is decomposable as a complete minimax-bettering ladder; this new condition, besides being weaker than the former, turned out to be both necessary and sufficient for the finite horizon Nash folk theorem.

Next, we state and prove a Nash folk theorem for finitely repeated games with unilateral commitments. This result. Theorem 11 is a variation of the main result in García-Jurado et al. (2000) to place it within our framework. More precisely, here we deal with utilities instead of with preferences, we allow for discounts, and we consider the set $F$ instead of the set $\{\varphi(a): a \in A\}$.

Theorem 1. Let $G=(N, A, \varphi)$ and let $v$ be its minimax payoff vector. Let $u \in F, u>v$. Then, for each $\varepsilon>0$, there are $\delta_{0} \in(0,1)$ and $T_{0} \in \mathbb{N}$ such that for each $\delta \in\left[\delta_{0}, 1\right]$ and each $T \geq T_{0}$, the game $U(G(\delta, T))$ has a Nash equilibrium payoff $w$ such that $\|w-u\|<\varepsilon$.

Proof. Let $G=(N, A, \varphi)$. Let $u \in F$ and let $\varepsilon>0$ be small enough so as to ensure that for each $i \in N, u_{i}-\varepsilon>v_{i}$. Since $u \in F$, there are $T^{\prime} \in \mathbb{N}$ and a sequence of action profiles $\left\{a^{t}\right\}_{t=1}^{T^{\prime}}$ such that $\left\|\frac{1}{T^{\prime}} \sum_{t=1}^{T^{\prime}} \varphi\left(a^{t}\right)-u\right\|<\frac{\varepsilon}{3}$. Now, by a continuity argument, there is $\delta^{\prime} \in(0,1)$ such that for each $\delta \in\left[\delta^{\prime}, 1\right],\left\|\frac{1-\delta}{1-\delta^{T^{\prime}}} \sum_{t=1}^{T^{\prime}} \delta^{t-1} \varphi\left(a^{t}\right)-u\right\|<\frac{2 \varepsilon}{3}$. Now, for each $T \in \mathbb{N}$ and each $\delta \in(0,1]$, let $\sigma^{*}$ be the strategy profile of $G(\delta, T)$ in which players successively play the actions in $\left\{a^{t}\right\}_{t=1}^{T^{\prime}}$; formally, $\sigma^{*}$ prescribes for each stage $k$, the action $a^{\bmod \left(k, T^{\prime}\right)}$, where $\bmod \left(k, T^{\prime}\right)$ is the remainder of the division of $k$ by $T^{\prime}$. Finally, there is $T^{\prime \prime} \in \mathbb{N}$ be such that for each $T \geq T^{\prime \prime}$ and each $\delta \in\left[\delta^{\prime}, 1\right],\left\|\varphi^{\delta, T}\left(\sigma^{*}\right)-u\right\|<\varepsilon$.

\footnotetext{
${ }^{4}$ Refer to Benoit and Krishna (1999) for a complete survey on the topic.
} 
Now, for each $\delta \in(0,1]$ and each $T \in \mathbb{N}$, let $G(\delta, T)=\left(N, S, \varphi^{\delta, T}\right)$. We define the following strategy profile $\left(\bar{S}^{c}, \bar{\alpha}\right)$ of $U(G(\delta, T))$ :

i) For each $i \in N, \bar{S}_{i}^{c}:=$ "If everybody plays according to $\sigma^{*}$ in the first stage, then I will play according to $\sigma^{*}$ forever".

ii) For each $i \in N$ and each $S^{c} \in \prod_{j \in N} 2^{S_{j}}$, we define $\bar{\alpha}_{i}\left(S^{c}\right)$ as follows:

- If $S^{c}=\bar{S}^{c}$ :

$-i$ plays according to $\sigma^{*}$ in the first stage.

- If everybody plays according to $\sigma^{*}$ in the first stage, then $i$ plays according to $\sigma^{*}$ forever.

- If in the first stage only player $j \neq i$ has deviated from $\sigma^{*}$, then, $i$ plays $\left(p_{-j}\right)_{i}$ forever.

- Otherwise, $i$ plays ad libitum.

- If $S^{c}=\left(S_{j}^{c}, \bar{S}_{-j}^{c}\right)$, where $j \neq i$ and $S_{j}^{c} \neq \bar{S}_{j}^{c}$ : $i$ plays $\left(p_{-j}\right)_{i}$ forever.

- Otherwise: $i$ plays ad libitum.

Now, since for each $T \geq T^{\prime \prime}$ and each $\delta \in\left[\delta^{\prime}, 1\right],\left\|\varphi^{\delta, T}\left(\sigma^{*}\right)-u\right\|<\varepsilon$, we also have that for each $i \in N, \varphi_{i}^{\delta, T}\left(\sigma^{*}\right)>u_{i}-\varepsilon>v_{i}$. The payoff of a deviating player $i$ in game $G(\delta, T)$ is bounded by $\frac{1-\delta}{1-\delta^{T}}\left(\mu_{i}\left(a_{-i}^{1}\right)+\sum_{t=2}^{T} \delta^{t-1} v_{i}\right)$. Hence, by increasing $T$ and $\delta$ we can also ensure that the latter payoff is as close to $v_{i}$ as we want. In particular, we can ensure that it is below $u_{i}-\varepsilon$.

Hence, we already have proved that there are $T_{0}$ and $\delta_{0}$ such that for each $\delta \in\left[\delta_{0}, 1\right]$ and each $T \geq T_{0}$, the strategy profile $\left(\bar{S}^{c}, \bar{\alpha}\right)$ is a Nash equilibrium of $U(G(\delta, T))$ whose payoff $w$ is such that $\|w-u\|<\varepsilon$.

Remark. Note that even if we work with the mixed extension of a given game, we can still define the sequence $\left\{a^{t}\right\}_{t=1}^{T^{\prime}}$ using pure strategies. By doing so, there is no need to play mixed actions in the equilibrium path constructed in the previous proof. Hence, whether we assume or not that mixed actions are observable does not affect the proof of Theorem [1.

Remark. If instead of writing $u \in F=\operatorname{co}\{\varphi(a): a \in A\}$ in the statement of Theorem [1] we write $u \in\{\varphi(a): a \in A\}$, then we can obtain an exact folk theorem instead of an approximate one. This is because if $u \in\{\varphi(a): a \in A\}$, then we can take an action $a \in A$ with payoff $u$ instead of the sequence whose payoff approximates $u$.

The main purpose for the rest of this Section is to state and prove a subgame perfect folk theorem with UC. The trick of the proof of Theorem [1 in which the strategies corresponding with many subgames were defined ad libitum, does not work for subgame perfection. Moreover, when dealing with UC, we face extremely large game trees. They have many subgames, some of which may correspond to senseless commitments. Thus, we need to use the VSPE concept instead of the classical SPE. Theorem 1 says that, when UC are possible, no condition is needed for the Nash folk theorem to hold. Note that the Nash equilibrium profile $\left(\bar{S}^{c}, \bar{\alpha}\right)$ defined in the proof of Theorem 1 is neither a SPE nor a VSPE; this is because, in general, the punishments to a player who deviates from the commitment are not credible. Now, Proposition 1 shows that not only the proof of Theorem 1 fails when we write VSPE instead of Nash equilibrium, but also the result itself is false.

Proposition 1. The counterpart of Theorem 1 for VSPE does not hold. 


\begin{tabular}{|c|c|c|}
\hline & L & $\mathrm{R}$ \\
\hline U & 10,11 & 1,10 \\
\hline$D$ & 11,0 & 0,1 \\
\hline
\end{tabular}

Figure 2: A counterexample for Proposition 1

Proof. We do the proof by means of an example. Let $G=(N, A, \varphi)$ be the game defined in Figure 2 The game $G$ does not have a Nash equilibrium. Moreover, $v=(1,1)$ and $\varphi(U, L)=(10,11)>v$. However, for each $T \in \mathbb{N}$ and each $\delta \in(0,1], U(G(\delta, T))$ does not have a VSPE. Suppose, on the contrary, that there are $\delta \in(0,1]$ and $T \in \mathbb{N}$ such that $\left(S^{c}, \alpha\right)$ is a VSPE of $U(G(\delta, T))$. If $S^{c}$ contains a unique element, then one of the players can change his commitment to no commitment at all (i.e., $S_{i}^{c}=S_{i}$ if $i$ is such a player) and deviate from the strategy in the final stage. Hence, there is a last stage in which, according to the path defined by $\left(S^{c}, \alpha\right)$, one of the players is free to play any action. Let $t$ be that stage and assume that, following the path of $\left(S^{c}, \alpha\right)$, player 1 can play both $U$ and $D$ at stage $t$ (a similar reasoning can be done if, instead, we take player 2). Moreover, let $x^{*}$ be the corresponding single-node of $G(\delta, T)$.

Now, let player 2 deviate to the strategy $\left(\bar{S}_{2}^{c}, \bar{\alpha}_{2}\right)$ defined as follows: (i) $\bar{S}_{2}^{c}:=$ "from stage $t+1$ on, I play according to the path defined by $\left(S^{c}, \alpha\right)$ " and (ii) for each $\hat{S}_{1}^{c} \in$ $2^{S_{1}}, \bar{\alpha}_{2}\left(\hat{S}_{1}^{c}, \bar{S}_{2}^{c}\right):=\alpha_{2}\left(S^{c}\right)$. Let $y$ be the single-node reached after $\left(S_{1}^{c}, \bar{S}_{2}^{c}\right)$ is played. By definition, $U(G(\delta, T))_{y}$ is a relevant subgame. Let now player 1 deviate, in $U(G(\delta, T))_{y}$, to the strategy $\bar{\alpha}_{1}$ defined as follows: for each $\hat{S}_{2}^{c} \in 2^{S_{2}}, \bar{\alpha}_{1}\left(S_{1}^{c}, \hat{S}_{2}^{c}\right):=\alpha_{1}\left(S^{c}\right)$. The subgame $U(G(\delta, T))_{y}$ is such that, when playing according to $\left(\bar{\alpha}_{1}, \bar{\alpha}_{2}\right)$, the single-node $x^{*}$ of $G(\delta, T)$ is reached again at stage $t$. Hence, the subgame beginning at the corresponding singlenode of $U(G(\delta, T))$, namely $x$, is relevant for $\left(S^{c}, \alpha\right)$. According to the commitments, both players can choose their two actions at $x$ and, from the stage $t+1$ on, player 2 's actions are determined by the commitment. Now, it is immediate to check that the relevant subgame $U(G(\delta, T))_{x}$ does not have any Nash equilibrium. Hence, $\left(S^{c}, \alpha\right)$ cannot be a VSPE.

In the counterexample we used in the proof above, we defined a game $G$ with no Nash equilibrium. Moreover, for each $T \in \mathbb{N}$ and each $\delta \in(0,1]$, the game $G(\delta, T)$ did not have any Nash equilibrium. On the other hand, we have the following positive result concerning the existence of VSPE for games with UC.

Proposition 2. Let $G=(N, A, \varphi)$ and let $\bar{a} \in A$ be a Nash equilibrium of $G$. Then, the game $U(G)$ has a $\operatorname{VSPE}\left(\bar{A}^{c}, \bar{\alpha}\right)$ with payoff $\varphi(\bar{a})$.

Proof. Let $\left(\bar{A}^{c}, \bar{\alpha}\right)$ be such that for each $i \in N$, we have

i) $\bar{A}_{i}^{c}=\left\{\bar{a}_{i}\right\}$,

ii) for each $j \neq i$ and each $A_{j}^{c} \in 2^{A_{j}}, \bar{\alpha}_{i}\left(\bar{A}_{-j}^{c}, A_{j}^{c}\right)=\bar{a}_{i}$. Finally, for each $A_{i}^{c} \in 2^{A_{i}}$, $\bar{\alpha}_{i}\left(\bar{A}_{-i}^{c}, A_{i}^{c}\right)=\hat{a}_{i}$, where $\hat{a}_{i} \in \operatorname{argmax}_{a_{i} \in \bar{A}_{i}^{c}}\left\{\varphi_{i}\left(\bar{a}_{-i}, a_{i}\right)\right\}$.

It is immediate to check that each such strategy profile $\left(\bar{A}^{c}, \bar{\alpha}\right)$ is a VSPE of $U(G)$.

In view of Proposition 2 it is clear that every Nash folk theorem for finitely repeated games can be easily adapted to provide a subgame perfect folk theorem for finitely repeated games with UC. More precisely, the necessary and sufficient condition for the Nash folk theorem in González-Díaz (2006), "that the game is decomposable as a complete minimaxbettering ladder", is a sufficient condition for the VSPE folk theorem with UC. The former condition implies among other things, the existence of a Nash equilibrium in the stage game 
$G$. Indeed, if the game $G$ does not have any Nash equilibrium, then none of the finitely repeated games $G(\delta, T)$ has (because of the last stage). We show in Example 2 that, even if the game $G$ does not have any Nash equilibrium, we can still have existence of VSPE in the finitely repeated game with UC.

Example 2. Let $G=(N, A, \varphi)$ be the game where $A_{1}=\{U, D\}, A_{2}=\{L, M, R\}$, and whose payoffs are defined in Figure 3

\begin{tabular}{c|c|c|c|}
\multicolumn{1}{c}{$\mathrm{L}$} & \multicolumn{1}{c}{$\mathrm{M}$} & \multicolumn{1}{c}{$\mathrm{R}$} \\
\cline { 3 - 5 } $\mathrm{U}$ & 10,10 & 0,1 & 1,0 \\
\cline { 2 - 4 } $\mathrm{D}$ & 11,0 & 1,1 & 0,2 \\
\cline { 2 - 4 } & &
\end{tabular}

Figure 3: A game without Nash equilibria

The game $G$ does not have a Nash equilibrium. Moreover, $v=(1,2)$. Now, for each $T \in \mathbb{N}$ and each $\delta \in(0,1]$, the payoff $(10,10)$ can be supported by a VSPE of $U(G(\delta, T))=$ $\left(N, S^{U}, \varphi^{U}\right)$. To check this assertion, consider the strategy profile $\left(\bar{S}^{c}, \bar{\alpha}\right)$ of $U(G(\delta, T))$ defined as follows: (i) $\bar{S}_{1}^{c}:=$ "I play $U$ in every stage", (ii) $\bar{S}_{2}^{c}:=$ "I never play $R$ ", and (iii) for each $i \in\{1,2\}$ and each $S_{i}^{c} \in 2^{S_{i}}, \bar{\alpha}\left(\bar{S}_{-i}^{c}, S_{i}^{c}\right)$ consists of playing, at each stage, the unique Nash equilibrium of the corresponding one stage game. Then, $\left(\bar{S}^{c}, \bar{\alpha}\right)$ is a VSPE and $\varphi^{U}\left(\bar{S}^{c}, \bar{\alpha}\right)=(10,10)$.

Proposition 2 says that we can use the UC to make actions credible, even actions that in the original game could be dominated. On the other hand, Example 2 shows that we can use the UC to go further than that. Hence, some more research is needed to find new sufficient conditions for the VSPE folk theorem. Now, we present a new theorem, a result which, with the aid of Proposition 2] is straightforward.

Theorem 2. Let $G=(N, A, \varphi)$ and let $v$ be its minimax payoff vector. Let $u \in F, u>v$. Then, for each $\varepsilon>0$, there are $\delta_{0} \in(0,1)$ and $T_{0} \in \mathbb{N}$ such that for each $\delta \in\left[\delta_{0}, 1\right]$ and each $T \geq T_{0}$, the game $U(U(G(\delta, T)))$ has a VSPE with payoff $w$ such that $\|w-u\|<\varepsilon$.

Proof. Immediate from the combination of Theorem 10 and Proposition 2

Theorem 2 implies that, when two stages of commitments are possible, any feasible and individually rational payoff of the original game can be achieved as a VSPE of the repeated game with UC. No assumption is needed for the original game, not even the existence of a Nash equilibrium.

Next, we briefly discuss the impact of Theorem 2 within the delegation framework discussed in the Introduction. First, from the point of view of our model with UC, the game $U(U(G(\delta, T)))$ can be difficult to motivate. It is true that we get a very strong result for the set of equilibrium payoffs of this game, but the fact that we allow for commitments on commitments might have unnatural features in some models. The point is that, when we introduced UC, we emphasized the fact that they were unilateral, i.e., the commitments of one player could not be conditional on the other players' commitments; if we allow for two stages of commitments, then we are indirectly allowing for commitments on commitments, and hence, we achieve the same payoffs we could get with a cooperative model. On the other hand, if we reassess the delegation situation corresponding with our UC model, and we do it in a similar way to that in the Introduction, then we have the following interpretation for the two stages of commitments. Consider a situation in which two firms are engaged in a competitive situation. Initially, the players are the presidents, and hence, in the first stage each president signs a contract with his principal in which the latter is committed not 
to play certain strategies and he will be paid proportionally to the payoff he finally gets. Then, in a second stage, a similar contract is signed between each principal and his agent. Finally, the agents play the original game but honoring the commitments. This situation has some important differences with the one stage situation: (i) the commitments that the president includes in the contract in the first stage can take into account the commitments that the principal will make with the agent at stage two, i.e., the contract between each president and his principal also commits the latter on the commitments he can sign with his agent, (ii) in the second stage the principals, being consistent with the commitments of their contract with the principals and in view of the commitments made by the rivals, choose a new commitment for the agents, and (iii) finally, the agents have to play being consistent with all the previous commitments. The hierarchical delegation model we have just described is quite natural and it is not difficult to think of real life situations with these sub-delegation structures. Hence, if such situations also correspond to some repeated game, then Theorem 2 says that, regardless of the properties of the underlying stage game, the "cooperative" (collusive) payoffs can be supported as a VSPE in the game with two stages of commitments.

\section{Final Remarks}

\section{Infinitely Repeated Games}

Although we have not formally introduced the model with infinitely repeated games, all the definitions can be immediately extended to encompass also this family of games; basically, replacing $T$ by $\infty$ in the definition of history and in the subsequent ones. Now, within this new framework, Proposition 2 still carries over. Now, recall that the classic Nash folk theorem for infinitely repeated games (see, for instance, Fudenberg and Maskin (1986)) states that, if the discount is close enough to 1 , every feasible and individually rational payoff can be approximated as a Nash equilibrium of the infinitely repeated game. Hence, if we combine this classic result with Proposition 2 we get the following Corollary:

Corollary 1. Let $G=(N, A, \varphi)$ and let $v$ be its minimax payoff vector. Let $u \in F, u>v$. Then, for each $\varepsilon>0$, there is $\delta_{0} \in(0,1)$ such that for each $\delta \in\left[\delta_{0}, 1\right]$ the game $U(G(\delta, \infty))$ has a VSPE with payoff $w$ such that $\|w-u\|<\varepsilon$.

Proof. It is immediate from the combination of Proposition 2 with the classic Nash folk theorem for infinitely repeated games.

\section{The State of Art}

Table 1 summarizes the results we have proved in this paper along with the classic folk theorems for repeated games with complete information. In particular, it shows the strength of Proposition 2 that allows to obtain many folk theorems for repeated games with UC as immediate corollaries of the classic ones. Hence, by looking at Table 1 one easily understands the strength of UC within this framework. Note that all the cells in the Table contain necessary and sufficient conditions, all of them but the one corresponding with the virtual subgame perfect folk theorem for finitely repeated games with UC; some more research is still needed concerning this case.

\section{Conclusions}

In this paper we have deepened in the literature of commitments. More specifically, we have studied the impact of UC in the folk theorems for repeated games. 


\begin{tabular}{|c|c|c|c|}
\hline & Without UC & 1 stage of UC & $\begin{array}{l}2 \text { stages } \\
\text { of UC }\end{array}$ \\
\hline $\begin{array}{l}\text { Nash Theorem } \\
\text { Infinite Horizon }\end{array}$ & $\begin{array}{c}\text { None } \\
\text { (Fudenberg and Maskin, 1986) }\end{array}$ & $\begin{array}{l}\text { None } \\
\text { (Prop. } 2\end{array}$ & $\begin{array}{l}\text { None } \\
\text { (Prop. [2) }\end{array}$ \\
\hline $\begin{array}{l}\text { (Virtual) Perfect Th. } \\
\text { Infinite Horizon }\end{array}$ & $\begin{array}{c}\text { Non-Equivalent Utilities } \\
\text { (Abreu et al., 1994) }\end{array}$ & 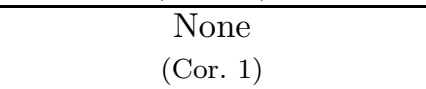 & $\begin{array}{l}\text { None } \\
\text { (Prop. [2) }\end{array}$ \\
\hline $\begin{array}{l}\text { Nash Theorem } \\
\text { Finite Horizon }\end{array}$ & $\begin{array}{c}\text { Minimax-Bettering Ladder } \\
\text { (González-Díaz, 2006) }\end{array}$ & $\begin{array}{c}\text { None } \\
\text { García-Jurado et al., 2000) }\end{array}$ & $\begin{array}{l}\text { None } \\
\text { (Prop. [2) }\end{array}$ \\
\hline $\begin{array}{l}\text { (Virtual) Perfect Th. } \\
\text { Finite Horizon }\end{array}$ & $\begin{array}{c}\text { Recursively-distinct } \\
\text { Nash payoffs (Smith, 1995) }\end{array}$ & $\begin{array}{c}\text { Minimax-Bettering Ladder } \\
\text { (Prop. \ only sufficient) }\end{array}$ & $\begin{array}{l}\text { None } \\
\text { (Th. 2) }\end{array}$ \\
\hline
\end{tabular}

Table 1: Necessary and Sufficient conditions for the folk theorems

We want to emphasize again the following fact. Because of the way we have modeled UC, it could seem that they are very far from the more standard models of commitment via delegation. But, as we pointed out in the Introduction and in the discussion of Theorem 2 UC can be used to model situations in which there is a principal who signs a contract with his agent with three features: (i) The agent has committed not play certain strategies, (ii) among the remaining ones his payoff is proportional to that of the principal, i.e., the agent can be thought of as a shareholder of the firm, and (iii) contracts are enforceable (everything is verifiable).

Moreover, we have shown that UC have very strong implications within the literature of repeated games with complete information. They lead to new folk theorems in which the assumptions needed for the classic results have been notably relaxed. Although we have presented our main results for pure strategies, we have also shown that everything can be extended to models with mixed strategies (without assuming observability of mixed actions). Moreover, as we pointed out in the remarks of Theorem 1 we can present exact folk theorems (as opposed to approximated) just replacing the set feasible set $F$ with $\{\varphi(a): a \in A\}$.

Finally, there are several open questions that should be tackled in the future. One of them is to refine the conditions for the finite horizon perfect folk theorem with UC. There is another important issue where some research is needed: the impact of UC in repeated games with incomplete information.

\section{References}

Abreu, D., P. K. Dutta, And L. Smith (1994): "The Folk Theorem for Repeated Games: A NEU Condition," Econometrica, 62, 939-948.

Benoît, J.-P. And V. Krishna (1987): "Nash Equilibria of Finitely Repeated Games," International Journal of Game Theory, 16, 197-204. 9902001.

(1999): "The Folk Theorems for Repeated Games: A Synthesis," EconWPA-

Caillaud, B., B. Jullien, And P. Picard (1995): "Competing Vertical Structures: Precommitment and Renegotiation," Econometrica, 63, 621-646.

Faí̃̃a-Medín, A., I. García-Jurado, J. Méndez-Naya, and L. MÉndez-Naya (1998): "Unilateral Commitments in the Finitely Repeated Prisoners' Dilemma," Annals of Operations Research, 84, 187-194. 
Fershtman, C. (1985): "Managerial Incentives as a Strategic Variable in a Duopolistic Environment," International Journal of Industrial Organization, 3, 245-253.

Fershtman, C., K. L. Judd, And E. Kalai (1991): "Observable Contracts: Strategic Delegation and Cooperation," International Economic Review, 32, 551-559.

FudenberG, D. And E. Maskin (1986): "The Folk Theorem in Repeated Games with Discounting or with Incomplete Information," Econometrica, 54, 533-554.

García-Jurado, I., L. Méndez-Naya, and F. Patrone (2000): "Unilateral Commitments in Finitely Repeated Games," International Game Theory Review, 2, 129-139.

GonzÁlez-DíAz, J. (2006): "Finitely Repeated Games: A Generalized Nash Folk Theorem," Games and Economic Behavior, 55, 100-111.

Kreps, D. M. And R. Wilson (1982): "Sequential Equilibria," Econometrica, 50, 863894.

Schelling, T. C. (1960): The Strategy of Conflict, Harvard University Press.

SElten, R. (1965): "Spieltheoretische Behandlung eines Oligopolmodells mit Nachfrageträgheit," Zeitschrift für die gesamte Staatswissenschaft, 12, 301-324.

Sklivas, S. D. (1987): "The Strategic Choice of Managerial Incentives," RAND Journal of Economics, 18, 452-458.

Smith, L. (1995): "Necessary and Sufficient Conditions for the Perfect Finite Horizon Folk Theorem," Econometrica, 63, 425-430.

VAn Damme, E. (1991): Stability and perfection of Nash equilibria, London, UK: SpringerVerlag.

Vickers, J. (1985): "Delegation and the Theory of the Firm," Economic Journal, 95, 138-47. 